\title{
Automatic Signal Processing of Front Monitor Radar for Tunneling Machines
}

\author{
Toru Sato, Member, IEEE, Ken-ya Takeda, Takashi Nagamatsu, Toshio Wakayama, Iwane Kimura, and \\ Tetsuya Shinbo
}

\begin{abstract}
It is planned to install a front monitor impulse radar on the surface of the rotating drill of tunnelling machines in order to detect obstacles such as casing pipes of vertical borings. The conventional aperture synthesis technique can no more be applied to such cases because the radar image of a pipe does not constitute a hyperbola as is the case for linear scanning radars. We have developed a special purpose signal processing algorithm with the aid of the discrete model fitting method, which can be used for any pattern of scanning. The details of the algorithm are presented together with the results of numerical simulations and test site experiments.
\end{abstract}

Keywords-Target detection, tunneling mechine, subsurface radar data processing.

\section{INTRODUCTION}

Recent technical advances in the tunnelling machines has opened an economical and time-efficient way of constructing a tunnel. The technique has also been introduced to the cases of digging a tunnel beneath a road, where the open-cut method were usually employed.

One of anticipated dangers in this application is the casing pipes left in the ground after vertical borings. If a tooth of the drill of a tunnelling machine is caught by the casing pipe, it may break through the pavement of the road, which will result in a disaster. It is thus planned to install a radar near the outer edge on the front surface of the rotating drill of the tunnelling machine in order to monitor obstacles such as steel pipes in front of the drill.

The requirements for the signal processing system of the front monitor radar is 1) to automatically detect echoes from the obstacles, 2) to distinguish the echo from other isolated targets, and 3) to alert the operator when the drill approaches a pipe or a large obstacle so that the speed of the rotation can be slowed down in order to reduce the damage to the drill.

Since the radar scans along a circular path, the echo from a pipe appears not as a hyperbola, but as a double-peaked curve, preventing the use of conventional aperture synthesis technique.

We have developed a special-purpose nonlinear least-squaresfitting algorithm which detects a pipe as well as isolated targets in the radar echo. The basic idea is the same as the Discrete Model Fitting algorithm (hereafter referred to as DMF) we have developed[5]. DMF constructs the model of the target and adjusts the model parameters so that the estimated echo signals from the

Toru Sato is with the Department of Electronics and Communication, Kyoto University, Sakyo-ku, Kyoto 606-01, Japan

NTT Co. Ltd., Nakase 1-6, Mihama-ku, Chiba, 261, Japan

Mitsubishi Heavy Industries, Ltd, 2-5-1 Marunouchi, Chiyoda-ku, Tokyo 100, Japan

Mitsubishi Electric Corporation, 5-1-1 Ofuna, Kamakura-shi, Kanagawa 247, Japan

Department of Information Systems, Osaka Institute of Technology, 1-79-1

Kitayama, Hirakata-shi, Osaka 573-01, Japan

Komatsu Co. Ltd., Manda, Hiratsuka-shi, Kanagawa 254, Japan model best-fit the observed ones in a least-squares sense. The parameters to be fitted are the distance and offset of the pipe from the center of the drill axis, its effective diameter, and the propagation constant of the medium. The effective diameter is the diameter of a conductive pipe that gives the same magnitude to the echo in the reference medium parameters.

After the principle of DMF method is briefly reviewed, the details of the algorithm including the preprocessing of data will be presented. The performance of the algorithm is examined using numerical simulations and test site experiments.

\section{Principle of Discrete Model Fitting Method}

The original DMF method reconstructs the image of the target assuming that it consists of a group of point targets with variable reflectivity. For a two-dimensional monostatic case, the electric field of the received signal at wavenumber $k$ and antenna position $r$ is expressed as

$$
E(k, r)=-\frac{k^{2}}{16} \sum_{i=1}^{N} \gamma_{i} H_{0}^{(1)}\left(k\left|\boldsymbol{r}-\boldsymbol{r}_{i}\right|\right)^{2},
$$

where $N$ is the number of point targets, $\gamma_{i}$ and $\boldsymbol{r}_{i}$ are the reflection coefficient and the position of the $i$-th target, respectively, and $H_{0}^{(1)}$ is Hankel function. For a given set of the model, the error of the estimation is given by

$$
F=\sum_{j, l}\left(\left|e\left(t_{j}, \boldsymbol{r}_{l}\right)-e_{\mathrm{obs}}\left(t_{j}, \boldsymbol{r}_{l}\right)\right|\right)^{2},
$$

where $e(t, r)$ is Fourier transform of $E(k, r)$ within the bandwidth of the radar, and subscript obs represents the observed value.

The DMF method modifies $\gamma_{i}, \boldsymbol{r}_{i}$ and $N$ so that $F$ becomes minimum. Since $F$ is not linear with respect to the model parameters, a non-linear least-squares fitting method is used to minimize it. Here we adopt the modified Marquardt method[1].

In the present application, two types of targets are considered; namely, a conducting pipe or a point target. As explained later, the algorithm automatically decides which type of target is in its view, and treat it accordingly. Figure 1 shows the coordinates when the radar observes a pipe. The upper panel shows the front view, and the lower panel shows the side view. The parameters to be found are positional parameters $R_{\text {off }}$ and $D$, and the reflectivity $\gamma$ of the pipe. The position of the antenna $r$ is expressed in terms of the rotation angle $\theta$. Instead of using a theoretical expression as in Eq. (1), the received waveform for the model $e(t, r)$ is synthesized using the measured waveform at a test site with a proper band limitation. It is thus expressed as

$$
e(t, \theta)=A(\phi) \frac{\exp \left(-\alpha c_{\mathrm{u}} t_{0}\right)}{c_{\mathrm{u}} t_{0}} \gamma e_{0}\left(t-t_{0}\right),
$$




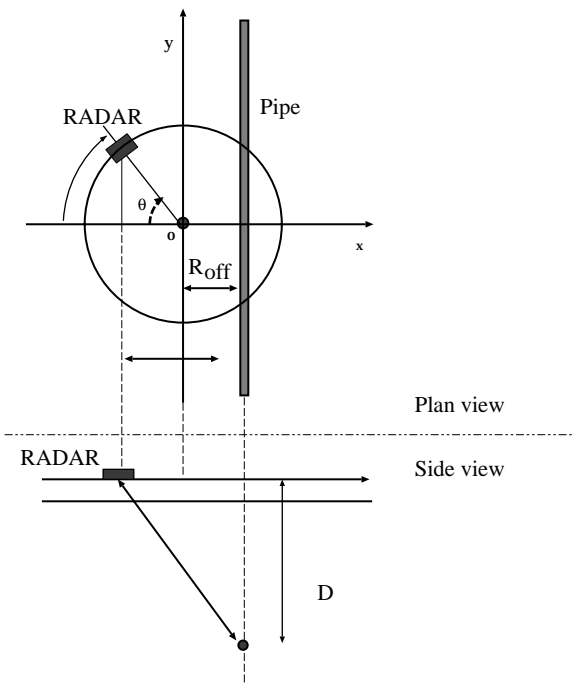

Fig. 1. Coordinates of the configuration.
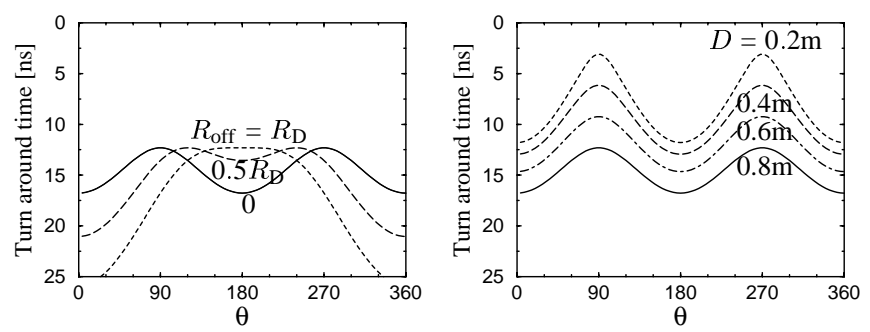

Fig. 2. Example of traces obtained from pipe echoes. Three curves in the left

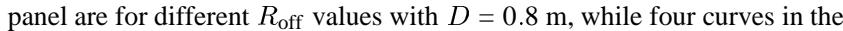
right panel are for different $D$ values with $R_{\text {off }}=0$.

where $A(\phi)$ is the two-way antenna pattern, $\alpha$ is the attenuation coefficient of the medium, $c_{\mathrm{u}}$ is the speed of the radio wave in the medium, $e_{0}(t)$ is the reference waveform of the echo from a pipe,

$$
\begin{aligned}
t_{0} & =\frac{2 \sqrt{\left(R_{\mathrm{D}} \cos \theta+R_{\mathrm{off}}\right)^{2}+D^{2}}}{c_{\mathrm{u}}} \\
\phi & =\tan ^{-1}\left(\frac{R_{\mathrm{D}} \cos \theta+R_{\mathrm{off}}}{D}\right)
\end{aligned}
$$

$R_{\mathrm{D}}$ is the radius of the antenna position from the drill center. Although the medium parameters $\alpha$ and $c_{\mathrm{u}}$ also need to be determined, they are estimated separately. Figure 2 shows examples of traces obtained from pipe echoes.

\section{Experimental ArRAngement}

In order to obtain basic data for designing the radar, we made a series of experiment at the test site rather than in the tunnel. Figure 3 shows a schematic sectional view of the test site. Considering that the tunneling machine pressurizes the cutting plane by water, the sand layer of the test site was covered by a water layer of 2-cm thickness. A steel pipe was embedded in the sand layer.

The actual drill surface of the tunneling machine on which the radar is planned to be installed has a diameter of $4 \mathrm{~m}$. Since

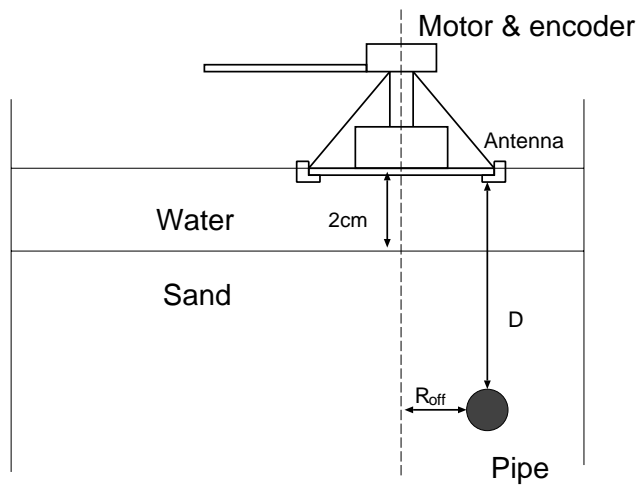

Fig. 3. Sectional view of the test site.

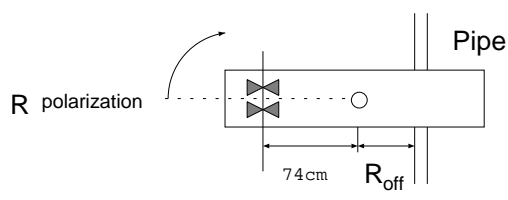

$\mathrm{L}$ polarization

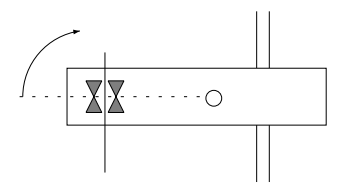

Fig. 4. Antenna arrangement of the radar.

it is not easy to realize this size in the test site, we prepared a rotating arm of $1.5 \mathrm{~m}$ long with two antennas mounted on its both ends ( $74 \mathrm{~cm}$ from the center). The two antennas are set with different polarizations referred to as 'radial' (R) and 'lateral' (L), respectively, as shown in Fig. 4.

The radar transmits mono-cycle pulses of $1 \mathrm{~ns}$ width at an interval of $3 \mu \mathrm{s}$. The peak output power is $72 \mathrm{~W}$. Received signal is sampled at $0.1 \mathrm{~ns}$ intervals up to $25 \mathrm{~ns}$, and digitized into 256 levels. 256 pulses transmitted while the arm rotates $2^{\circ}$ are digitally averaged, and stored on a disk.

\section{Data Processing of Each Image}

Data processing consists of two stages: 1) Processing of each image taken during a single rotation of the drill. Parameters estimated in this stage are $R_{\mathrm{D}}, D$, and $\gamma .2$ ) Processing of multiple images while the drill approaches the target. The medium parameters $c_{\mathrm{u}}$ and $\alpha$ are estimated in this stage. We describe the details of the first stage in this section, while the second stage is explained later.

\section{A. Polarization Effects}

Between the two available polarizations, the one appropriate for observing a pipe depends on the relative location of the pipe to the drill. Figure 5 shows the front view (the plan view at our test site) of the radar when it observes a pipe with the two polarizations. As can be easily seen, the radial polarization should be used when the pipe is encountered at the center of the drill $\left(\theta=90^{\circ}\right.$ and $\left.270^{\circ}\right)$, while the lateral polarization is suited when the drill picks the pipe at its outer edge $\left(\theta=0^{\circ}\right.$ and $\left.180^{\circ}\right)$. In or- 


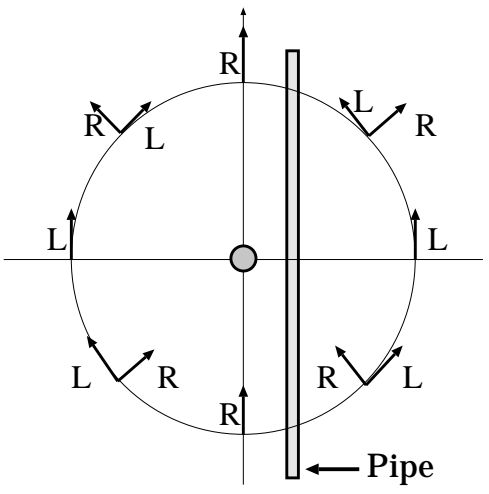

Fig. 5. Radial and lateral polarizations used for detecting a pipe.

der to make best use of the two polarizations, we combine them as follows:

$$
W=W_{R} \sin ^{2} \theta+W_{L} \cos ^{2} \theta
$$

where $W, W_{R}$, and $W_{L}$ are the combined, radial, and lateral waveforms, respectively.

\section{B. Subtraction of Clutter Echoes}

As is common for ground probing radars which receive the echo of the same polarization as transmitted, the received echoes are contaminated with strong clutters from the ground surface, interface between water and ground, and from layers in the ground. The conventional way is to subtract the mean signal level of the same depth for different antenna locations. Although it is in principle applicable to our case, it also suppresses the desired signal when the pipe is located on the outer edge of the drill, where the antenna is mounted. As shown in the case of $R_{\text {off }}=R_{\mathrm{D}}$ in Fig. 2, the turn around time stays roughly constant over a wide angular range near $180^{\circ}$. In such a case, the mean signal level at this depth does not correctly represent the background level in the absence of the echo. The mean value also differs from the true level of the clutter echoes if the desired signal has a comparable or stronger level than the clutter.

In this situation, an effective way of estimating the background clutter level is to take the mode, or the value which occurs most frequently, of the received signal of the same depth for all $\theta$ instead of the mean, because the echo from a pipe does not stay at a fixed depth for more than one fourth of the entire rotation even if pipe is located at the drill edge. The mode should then represent the level of the clutter echo only. However, the computation of the mode requires to compute the distribution function of the signal level, which may contain large statistical uncertainty when computed from a limited number of samples (180 samples for the current case). We thus employ the median level as a more stable estimate[2].

The advantage of using the median in place of the mean is that the former does not use the strength of the signal, so that the existence of a strong target does not affect the estimate of the clutter level. Figure 6 compares the median and the mean of a numerical example of random noise whose mean and the standard deviation are 0 and 1, respectively, plus a Gaussian signal with peak amplitude of 10 . While the estimated noise level by

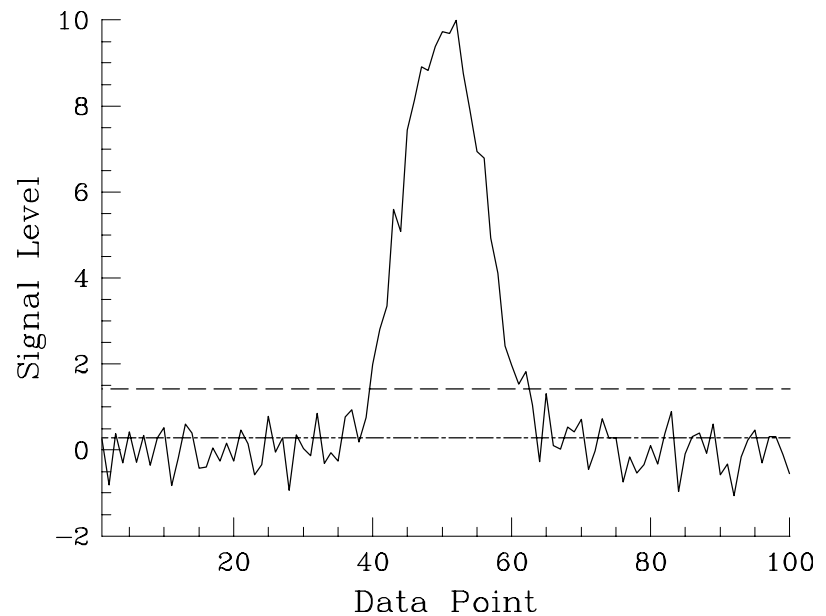

Fig. 6. Estimates of the background level of a random noise with a strong Gaussian signal by the median (solid line) and by the mean (dashed line).

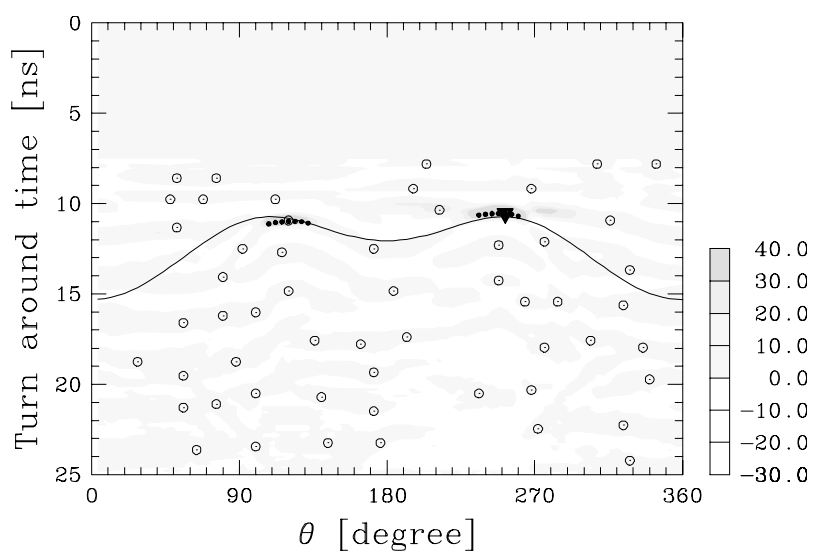

Fig. 7. Example of the received echo contour at the test site after polarization synthesis and subtraction of the median.

the mean is biased to 1.43 , the median gives a bias of only 0.29 .

Figure 7 shows an example of the test site data. The signal level is given as a contour diagram after polarization synthesis and subtraction of the median value. The resolution is $2^{\circ} \times 0.2 \mathrm{~ns}$ $(180 \times 128$ points in total $)$. The dark area shows strong positive signal level, while white areas indicate negative values.

\section{Detection of Peaks}

The next step is to find strong peaks in the contour, preferably a pair of two peaks located symmetrically with respect to $\theta=180^{\circ}$, which is the condition for peaks corresponding to a pipe. We first find all local maxima in the data, as shown by open circles in Fig. 7. A local maximum is defined as a point that gives the maximum signal intensity in the grid of $13 \times 13$ points centered at that point.

As clearly seen in this figure, most of these local maxima do not represent the desired echo from the pipe. We thus need to migrate these points using the characteristics of the echo. Here we use the fact that the desired echo should follow smooth curves as shown in Fig. 2. From each of these local maxima, whose posi- 
tion is $\left(\theta_{j 0}, t_{i 0}\right)$, we then try to extend a curve in the $\theta$-direction by connecting the maximum points in the $t$-direction at given $\theta$ [3]. From a curve point $\left(\theta_{i}, t_{j}\right)$, the next curve point at $\theta_{i+1}$ is determined as a point that has maximum signal intensity in the range from $\left(\theta_{i+1}, t_{j-5}\right)$ to $\left(\theta_{i+1}, t_{j+10}\right)$. The extension of the curve is terminated when the signal intensity on the curve becomes less than half of that at $\left(\theta_{i 0}, t_{j 0}\right)$. The curve is also extended to negative $\theta$-direction in the same manner.

The same procedure is repeated for the nearest local maximum from the conjugate point with respect to $\theta=180^{\circ}$. Note that the two peaks may coincide if the first peak is located near $\theta=180^{\circ}$. For the pair of curves which consists of $N$ points in total, the theoretical curve given by Eq. 4 is fitted. The 'badness' of the pair of curves is given by

$$
b=\frac{\sum_{i}\left(t_{j}-t_{\mathrm{fit}}\right)^{2}}{N^{2} W_{1} W_{2}},
$$

where $t_{\text {fit }}$ is the time delay of the best fit curve at $\theta_{i}$, and $W_{1}$ and $W_{2}$ are the signal intensity of the two peaks. The expression for $b$ was chosen empirically to give a small badness for a curve that has a long horizontal extension, strong echo intensity, and also a good fit to the theoretical curve.

Triangles plotted on Fig. 7 indicate the pair of peaks whose badness is minimum. The curves extended from the peaks are indicated by curves with dots. The smooth curve overlaid on the figure is the best fit theoretical curve.

The judgment on whether the detected echo comes from a pipe or an isolated target can be safely made by checking the existence of the accompanying echo at the conjugate position to the strongest one in the data. When a pair of echoes is detected, the angular separation between the pair is used to give the initial guess of the model parameters, while the peak without accompanying one should be used for the initial guess of the echo trace from an isolated single target.

\section{Antenna Pattern}

Before applying DMF, we need to determine the antenna pattern $A(\phi)$ in Eq. 3. Although the pattern can fairly easily determined from the observed variation of the echo intensity versus $\theta$, the effect of the attenuation should be removed. The attenuation coefficient of the medium is estimated from the difference in the echo intensity from successive rotations of the drill as will be discussed later. The left panel of Fig. 8 shows the measured variation of the echo intensity from a pipe located at $R_{\text {off }}=0$ versus $\theta$. The dashed curve shows the signal level to be received by an omnidirectional antenna estimated using the attenuation coefficient thus estimated and the distance between the antenna and the pipe at each angle. The ratio of the dashed and the solid curves thus gives the contribution of the directivity of the antenna.

After correction for the attenuation, this pattern versus $\theta$ is converted into the actual antenna pattern using Eq. 5, as plotted in the right panel of Fig. 8. The dashed curve in the right panel is the best fit Gaussian pattern, which will be used throughout in the DMF method. In the current algorithm, the antenna pattern is fixed to the one thus derived. It is possible, however, to modify the assumed antenna pattern on the real time as the drill proceeds if a clearer echo from a pipe or a point target is identified.
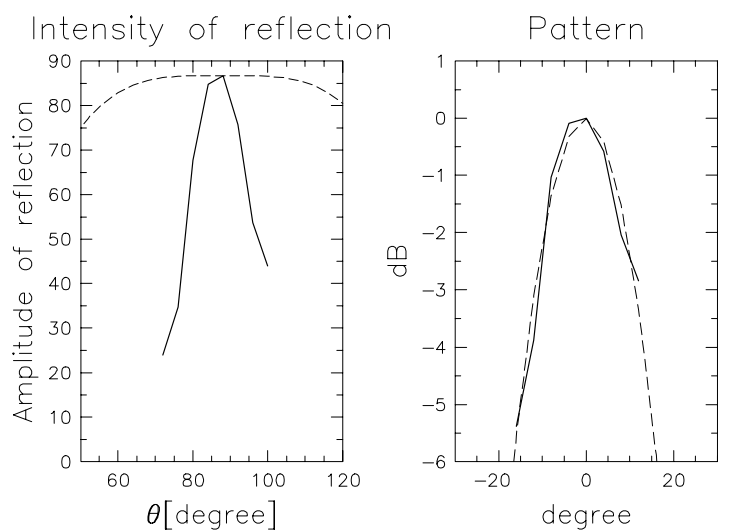

Fig. 8. Estimation of the antenna pattern. Solid curve in the left panel shows the measured intensity of the echo versus $\theta$, and dashed curve indicates the estimated attenuation. The right panel shows the antenna pattern derived from the data in the left panel. The dashed curve is the fitted Gaussian pattern.

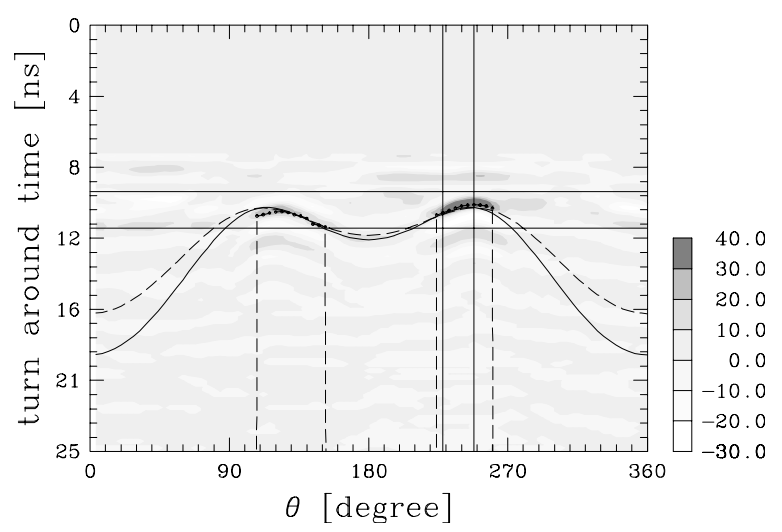

Fig. 9. An example of test site experiments which demonstrates the ambiguity in estimated $c_{\mathrm{u}}$. Solid and dashed curves show theoretical traces of a pipe fitted to data assuming $c_{\mathrm{u}}=5 \times 10^{7} \mathrm{~m} / \mathrm{s}$ and $7 \times 10^{7} \mathrm{~m} / \mathrm{s}$, respectively.

\section{Use of Multiple Rotations}

We have so far obtained necessary initial parameters for $R_{\text {off }}$, $D$, and $\gamma$ in Eqs. (3)-(5), which should be changed in the nonlinear fitting procedure. We have also determined the antenna pattern $A(\phi)$. The remaining parameters to be given are the medium parameters $c_{\mathrm{u}}$ and $\alpha$. Apparently, the attenuation coefficient $\alpha$ cannot be determined from measurements of targets with unknown reflectivity $\gamma$. It is thus necessary to estimate $\alpha$ separately.

The speed of radio wave $c_{\mathrm{u}}$ in the medium, or equivalently the dielectric constant of the medium, can be, in principle, determined in the fitting of each image described in the previous section. However, the accuracy of the estimated value is very poor when the attenuation of the medium is large. Figure 9 shows an experimental results with two theoretical curves for the echo trace from a pipe fitted to data assuming different values of $c_{\mathrm{u}}$. As is clear from the figure, both curves agree well with data in the region where the observed echo has a significant magnitude, although the estimated depth $D$ differs largely for these two cases. It is therefore difficult to determine $c_{\mathrm{u}}$ from a single data unless the attenuation is weak enough so that the echo trace can be 

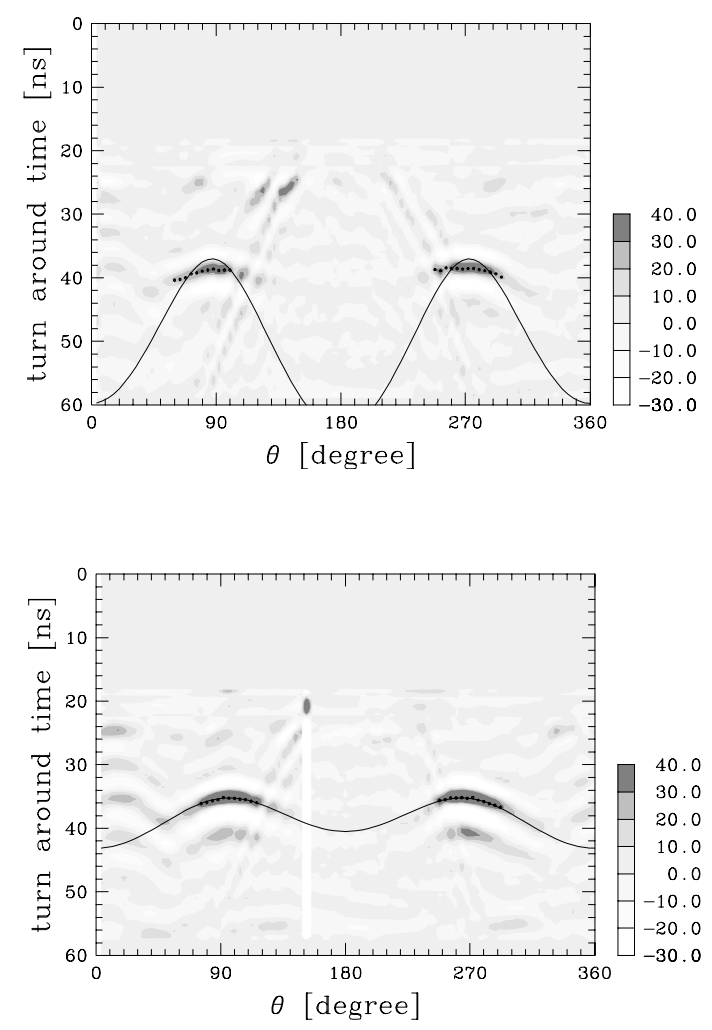

Fig. 10. Result of fitting with an inappropriate initial value of $c_{\mathrm{u}}$ (upper panel), and that at the third rotation (lower panel). The depth of the pipe is $100 \mathrm{~cm}$ and $90 \mathrm{~cm}$ for the first and the third rotations, respectively.

obtained from regions where the dependence of the trace on $c_{\mathrm{u}}$ becomes evident.

The major advantage of the front monitor radar compared to other subsurface radars is that a series of data can be obtained as the drill surface approaches to the target. Two or more sets of data taken during different cycles of drill rotation can be used to resolve the ambiguity in $\alpha$ and $c_{\mathrm{u}}$.

\section{A. Estimation of the Dielectric Constant of the Medium}

For the $m$-th rotation of a series of rotations in which the same pipe is detected, the minimum delay time $t_{0}$ at $\theta=$ $\cos ^{-1}\left(R_{\text {off }} / R_{\mathrm{D}}\right)$ is expressed as

$$
t_{m}=2\left(D_{1}-\Delta_{m}\right) / c_{\mathrm{u}},
$$

where $D_{1}$ is the distance of the pipe at the first rotation, and $\Delta_{m}$ is the progress of the drill from the first rotation to be measured by the stroke sensor. Note that $t_{m}$ in Eq. (8) is the parameter directly measured in each rotation, although it is formally expressed as $2 D / c_{\mathrm{u}}$. Eq. (8) is thus used to determine $D_{1}$ and $c_{\mathrm{u}}$ at the second rotation, and in a least-squares sense for each rotation with $m>$ 2. The intensity of the echo is used as the weighting factor in this fitting.

An arbitrary initial value is given for $c_{\mathrm{u}}$ at the first rotation. If this values is inappropriate, the theoretical trace determined in this rotation disagrees with data as shown in the upper panel of Fig. 10. The initial value of $c_{\mathrm{u}}$ given here is $3.0 \times 10^{7} \mathrm{~m} / \mathrm{s}$, while the true value determined from the measured delay of the echo and the known depth of the pipe is $6.6 \times 10^{7} \mathrm{~m} / \mathrm{s}$. Since $t_{m}$ value determined from Eq. (4), which corresponds to the peak of the theoretical trace, is a poor estimate of $t_{1}$, the peak of data with the same $\theta$ is used instead. The lower panel of Fig. 10 shows the result of fitting at $m=3$. The data used in this figure were taken at the test site with a pipe embedded at a variable depth of $D=100 \mathrm{~cm}$ to $80 \mathrm{~cm}$ at an interval of $5 \mathrm{~cm}$. In contrast to the poor fitting made at the first rotation, which is due to the false initial value of $c_{\mathrm{u}}$, the fitting improved significantly at the second rotation and the following ones. The speed of radio wave estimated using Eq. (8) after the second rotation is quite stable with variations of about $\pm 10 \%$.

We have assumed that the medium is uniform. It is likely that the medium is horizontally stratified with layeres having different dielectric constant across the diameter of the drill. The curves shown in Fig. 2 will then be distorted. In most cases, it may not seriously affect the estimate because the fitting is made to the entire tarce over one rotation of the drill. In some cases, however, the inhomogeneity will have a bad influence. The worst case will be that a layer with smaller (or larger) dielectric constant than the background is located around the point where the distance between the antenna and the pipe is minimum (such as $\theta=180^{\circ}$ for $R_{\text {off }}=R_{\mathrm{D}}$ ), which will make the peaks in the right panel of the figure more pronounced (or dumped) than in the uniform medium. Then the distance to the pipe will be incorrectly estimated. However, such a situation may be identified as drill proceeds because the estimated distance to the pipe will decrease faster (or slower) than the drill actually proceeds. The bias also approaches to zero as the drill approaches to the pipe.

\section{B. Estimation of the Attenuation Coefficient}

The attenuation coefficient $\alpha$ is estimated in a similar manner as $c_{\mathrm{u}}$. The apparent reflectivity of the pipe estimated at the $m$-th rotation assuming no attenuation is expressed as

$$
\gamma_{m}=\gamma \exp \left\{-2 \alpha\left(D_{1}-\Delta_{m}\right)\right\}
$$

where $\gamma$ is the true reflectivity of the target. After determining $D_{1}$ using Eq. 8, $\gamma$ and $\alpha$ is estimated by applying the linear least-squares fitting to $\log \gamma_{m}$. The attenuation coefficient of the medium of the data in Fig. 10 is estimated to be $7.4 \mathrm{~dB} / \mathrm{m}$.

It is thereby possible to estimate roughly the diameter of the pipe assuming that it is metallic. Even though the material of the pipe is not a metal, the estimate may serve at least as an 'effective diameter'. The information on the medium parameters $c_{\mathrm{u}}$ and $\alpha$, which can be converted into the permittivity and conductivity of the medium, may be useful in controlling the speed of the drill as well as for the hazard prevention.

We have implicitly assumed that the reference waveform $e_{0}(t)$ is constant for all rotations. Since the attenuation is frequency dependent, the band limitation effect on the waveform of the echoes from deeper targets should be taken into consideration[4]. Although it is in principle possible to include this effect in DMF, the current algorithm does not alter $e_{0}(t)$ after it is detected, because the range of interest is limited to a few to several tens of centimeters.

\section{Summary}

A special-purpose signal processing algorithm has been developed for detection of obstacles in front of the drill surface of 
a tunneling machine. The discrete model fitting (DMF) method was modified so that the echo from a vertical pipe can be identified in the data of a front monitor radar installed on the rotating surface of the drill. Algorithms for estimating necessary initial guesses for the model parameters have been presented. Medium parameters such as the attenuation coefficient and the dielectric constant of the medium were determined by making use of multiple data taken as the drill approaches to the target.

We are currently adjusting the various threshold levels used in the algorithm to give a reliable alarm to the drill operator based on the data taken at a test site in the laboratory. The alarm should consist of multiple levels that may give the information on whether a pipe or an isolated target is detected, distance to the target, and its effective size.

\section{ACKNOWLEDGMENT}

This work was supported in part by Grant-in-Aid for Scientific Research (A) 06555108 of the Ministry of Education, Science, Sports and Culture, Japan.

\section{REFERENCES}

[1] D. W. Marquardt, "An algorithm for least squares estimation of nonlinear parameters," J. Soc. Indust. Appl. Math., vol. 11, no. 2, pp. 431-441, 1963.

[2] T.A. Nodes, and N.C. Gallagher Jr., "Median filters: some modifications and their properties," IEEE Trans. Acoust., Speech Signal Process., vol. ASSP-30, no. 5, pp. 739-746, 1982.

[3] T. Sato, K. Takeda, T. Wakayama, I. Kimura, T. Abe, and T. Shinbo, "Automatic data processing procedure for ground probing radar," IEICE Trans. Commun., vol. E77-B, no. 6, pp. 831-837, 1994.

[4] G. Turner, "Propagation deconvolution," Proc. 4th Int. Conf. Ground Probing Radar, pp. 85-93, 1992.

[5] T. Wakayama, T. Sato, and I. Kimura, "High-resolution radar image reconstruction using an arbitrary array," IEICE Trans. Commun., vol. E76-B, no. 10 , pp. $1305-1312,1993$.

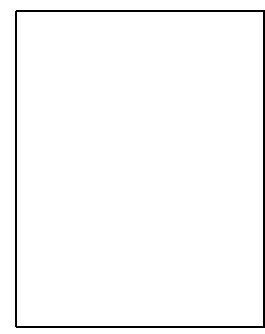

Toru Sato received the B.E., M.E., and Ph.D. degrees in electrical engineering from Kyoto University, $\mathrm{Ky}$ oto, Japan in 1976, 1978, and 1982, respectively. He has been a staff of Kyoto University since 1983, and is now an associate professor at Graduate Scool of Electronics and Communication Engineering. His research interests have been radar remote sensing of the atmosphere, observations of precipitation using radar and satellite signals, radar observation of space debris, signal processing for subsurface radars, and digital satellite communication. He was awarded Tanakadate Prize in 1986. He is a member of IEEE, American Meteorological Society, Institute of Electronics, Information and Communication Engineers, the Japan Society for Aeronautical and Space Sciences, and the Society of Geomagnetism and Earth, Planetary and Space Sciences.

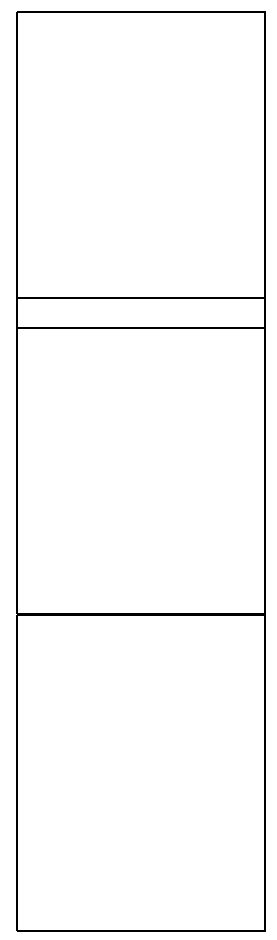

Kenya Takeda received the B.E. and M.E. degrees in electrical engineering from Kyoto University, Japan in 1992 and 1994, respectively. He joined NTT Co. Ltd in 1994. He is a member of Institute of Electronics, Information and Communication Engineers.

Takashi Nagamatsu received the B.E. and M.E. degrees in electrical engineering from Kyoto University, Japan in 1994 and 1996, respectively. He joined Mitsubishi Heavy Industries Ltd in 1996. He is a member of Institute of Electronics, Information and Communication Engineers.

Toshio Wakayama received the B.E., M.E., and Ph.D degrees in electrical engineering from Kyoto University, Japan in 1990, 1992, and 1995, respectively. He joined Mitsubishi Electric Corporation in 1995. His research interest is radar system design and radar signal processing. He is a member of Institute of Electronics, Information and Communication Engineers.

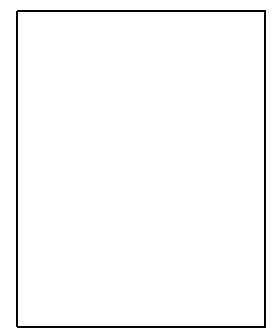

Iwane Kimura received the B.E., M.E., and Ph.D. degrees in electrical engineering from Kyoto University, Kyoto, Japan in 1955, 1957, and 1961 respectively. Since 1960, he has been a staff of Kyoto University and has been a Professor from 1971 to 1996 . He is now a Professor of Department of Information Systems, Osaka Institute of Technology, and also is a Professor Emeritus of Kyoto University. He has also been a visiting Professor of Institute of Space and Astronautical Science from 1981 to 1991 . From 1964 to 1965 , he was a research associate at Radioscience Laboratory, Stanford University on leave from Kyoto University. His research interests have been in radio science; particularly remote-sensing of upper atmospheres by radar techniques, and space plasma physics, such as theoretical study on propagation and generation of radio waves in magnetospheric and ionospheric plasmas, plasma measurements by rocket-Doppler technique, and active wave experiments in space plasmas using scientific satellites. He was awarded Inada Memorial Prize in 1958, and Tanakadate Prize in 1961. He is a member of Institute of Electronics, Information and Communication Engineers, the Institute of Electrical Engineers of Japan, the Society of Geomagnetism and Earth, Planetary and Space Sciences, and the American Geophysical Union.

Tetsuya Shinbo received the B.E. and M.E. degrees in electrical engineering from Kanazawa University, Japan in 1985 and 1987, respectively. He joined Komatsu Co., Ltd. in 1987. His research interest is detection of underground objects using radio wave. He is also working now for the Ph.D. degree at Department of Electrical Engineering, Kanazawa University. 